\title{
Quelle place pour l'oral dans les centres de littératie?
}

\author{
EMMANUELLE SOUCY \\ Université du Québec en Outaouais
}

\section{Résumé}

Les centres de littératie sont un dispositif d'enseignement-apprentissage qui permet l'enseignement et l'apprentissage de la lecture, de l'écriture ainsi que de la communication orale (Diller, 2010; Nations et Alonso 2001; Owocki, 2005; Swartz et all, 2003). Toutefois, on en sait bien peu sur l'enseignement et l'apprentissage de l'oral dans ces centres. À l'aide d'une recherche exploratoire, nous décrivons la place et le statut de l'oral dans ces centres dans une classe de deuxième année du primaire à partir de l'entrevue de deux enseignantes et d'observations dans une classe.

Mots-clés (4)

Centres de littératie, enseignement, oral, primaire

\section{Introduction}

L'importance de l'oral dans le développement des habiletés de littératie n'est plus à nier. La littératie se définit comme étant une compétence générale, qui désigne l'interaction de différentes compétences qui permettront de mieux comprendre, d'utiliser et d'interpréter l'information contenue dans différents textes, qu'ils soient imprimés, oraux ou médiatiques (Buors et Lentz, 2009 ; Dumais, Soucy et Plessis-Bélair, accepté).

Soucy (2016, p. 7) définit le volet oral de la littératie comme étant «l'ensemble des interactions ayant lieu en milieu scolaire ou extrascolaire, incluant la production et la compréhension orales, l'interprétation critique de messages, de même que les capacités d'écoute et d'échange ainsi que l'importance de la prise en compte de soi, de l'autre et du contexte pour se dire et se construire ". L'importance des habiletés requises par ce volet oral de la littératie est manifeste. Tout d'abord, elles ont une influence sur la réussite des élèves en lecture et en écriture (Bianco, 2015). Ensuite, elles servent aussi de levier important pour développer leur plein potentiel (Dumais, 2015). Lafontaine et Pharand (2015) affirment que les compétences en littératie sont requises pour réussir dans une société basée sur le savoir. Il est donc important et pertinent de développer le langage oral et d'enseigner des stratégies de communication orale, particulièrement au $1^{\mathrm{er}}$ cycle du primaire, afin de former des locuteurs capables de s'exprimer dans toutes situations de la vie en classe et hors de l'école, en plus de leur donner les bases nécessaires à l'apprentissage de la lecture et de l'écriture.

Cet article présentera d'abord la problématique et la question de recherche. Ensuite, un cadre conceptuel définira les centres de littératie ainsi que les statuts de l'oral. Les objectifs poursuivis par cette recherche seront formulés. Par la suite, dans la section méthodologie, le type de recherche ainsi que l'échantillon seront présentés, suivis des outils et des méthodes de collecte de données et d'analyse. Enfin, une section présentera 
les résultats des deux objectifs de la recherche ainsi qu'une discussion pour les interpréter. La conclusion fera état des limites de la recherche ainsi que des pistes de recherche future.

\section{Problématique}

Plusieurs chercheurs se sont intéressés à l'enseignement de au cours de la dernière décennie (Dumais et Lafontaine, 2011 ; Lafontaine, Plessis-Bélair et Bergeron, 2007 ; Simard, Dufays, Garcia-Debanc et Dolz, 2010). Malgré tout, les enseignants relèguent encore l'oral au dernier plan (Sénéchal et Chartrand, 2012). Considèrent-ils qu'en intégrant davantage l'enseignement de l'oral en classe, ils devront nécessairement le faire au détriment des autres matières ? Il est possible de le croire, sachant que la plupart des enseignants se disent préoccupés par le manque de temps (Maurer, 2001 ; Simard et al. 2010).

Depuis déjà quelques années, des professionnels de l'enseignement font état d'un dispositif $d^{\prime}$ 'enseignement-apprentissage ${ }^{1}$ qui permet une organisation efficace de l'enseignement de la langue. Il s'agit des centres de littératie, définis dans la prochaine section, qui permettent l'apprentissage de la lecture, de l'écriture et de la communication orale (Diller, 2010 ; Nations et Alonso 2001; Owoki, 2005 ; Swartz et al., 2003). Présent surtout du côté anglophone, il en existe aussi au Québec. Il a été permis d'en constater la présence dans quelques écoles, sans toutefois que cela ne soit documenté. De plus, il semble y avoir un vide théorique au sujet de la présence de l'oral dans ces centres. En effet, aucun écrit, ni scientifique ni professionnel, ne fait ressortir clairement la manière dont l'oral y est enseigné et utilisé, et comment sont effectués les apprentissages par les élèves. Considérant le volet oral comme un élément important des compétences de littératie (Dumais et al., accepté), il semble important d'apporter quelques éléments de réponses et d'en savoir davantage sur l'oral dans les centres de littératie. Ainsi, la question de la présente recherche est la suivante : quelle est la place de l'oral au sein de centres de littératie?

\section{Cadre conceptuel}

Le cadre conceptuel s'appuie sur les centres de littératie ainsi que les deux statuts de l'oral, c'est-à-dire l'oral médium d'enseignement et l'oral objet d'enseignement. .

\section{Les centres de littératie}

Les centres de littératie sont définis par plusieurs auteurs comme étant un dispositif d'enseignement-apprentissage de la langue qui propose une approche concrète de la littératie (Fleminton, Hewins, Proulx et Villiers, 2012 ; Hodges et McTigue, 2014 ; Kracl ; 2012, Rybczynski et Troy, 1995 ; Stone, 1996 ; Stout, 2009 ; Swanson, 2013). Le travail en centres de littératie, surtout utilisé au primaire, permet l'immersion quotidienne dans la littératie en offrant une variété d'activités de lecture, d'écriture, d'écoute et de communication à faire seul ou en petit groupe. C'est cette variété d'expériences qui donne de vraies raisons de lire, d'écrire, de discuter et d'écouter qui permet aux élèves de

\footnotetext{
${ }^{1}$ Un dispositif d'enseignement-apprentissage consiste en un ensemble cohérent qui inclut tout autant les ressources matérielles et humaines, que les stratégies, les méthodes et les acteurs qui interagissent dans un contexte donné (Rosselle, 2013).
} 
développer leurs compétences en littératie.

Ainsi, en offrant de larges blocs de littératie ${ }^{2}$, l'enseignant peut davantage équilibrer les moments d'enseignement-apprentissage en grand groupe et ceux qui sont guidés ainsi que les moments d'apprentissage autonome (Russell, 2014). Ces moments de travail autonome doivent être aussi efficaces que le temps passé avec l'enseignant. C'est pourquoi il est important que les élèves apprennent à travailler sans l'aide de l'enseignant, tout en poursuivant et en organisant leurs propres apprentissages (Ford et Opitz, 2002 ; Fountas et Pinnel, 1996 ; Hodges et McTigue, 2014 ; Kracl, 2012). Pour développer l'autonomie à l'intérieur des périodes réservées aux centres de littératie, plusieurs auteurs recommandent de modeler tant les comportements attendus que les tâches proposées (Russell 2014 ; Stout, 2009 ; Swanson, 2013 ; Worthy et al., 2015).

Des minileçons d'une durée d'environ 7 à 10 minutes sont faites en grand groupe. Lors de ces minileçons, un enseignement spécifique sera ciblé : l'explication d'une règle de grammaire, le recours à une stratégie de lecture, la manière de se comporter dans les centres, la façon d'utiliser le matériel disponible ou les consignes pour réaliser les tâches constituent quelques exemples d'objets de minileçons. Certains auteurs (Ford et Opitz, 2002 ; Russell 2014) proposent une manière de fonctionner qui pourrait se résumer ainsi :

- Nommer l'objectif de la leçon ;

- Expliquer ce dont il s'agit ;

- Faire une démonstration ou un modelage ;

- Proposer une application directe de ce qui a été modelé.

Après cette entrée en matière, les élèves sont invités à se rendre dans différents centres où ils travailleront pour une durée variant de 20 à 30 minutes. C'est à ce moment que l'enseignant travaillera avec un sous-groupe d'apprenants, pour approfondir les apprentissages, selon leurs besoins particuliers. Une fois la période de 30 minutes terminée, l'enseignant reprend l'ensemble du groupe avec un nouvel enseignement, à la suite de quoi, les élèves se rendent dans un centre différent. Une fois dans les centres, les élèves travaillent de manière autonome, seuls ou en petits groupes. Lorsqu'ils travaillent en petits groupes, les élèves peuvent discuter, se questionner et s'entraider pour réaliser les tâches proposées. Ce déroulement se produira entre trois et quatre fois, lors d'un bloc d'environ 120 minutes.

Pendant les périodes de centres de littératie, l'enseignant communique oralement les objectifs, donne des consignes et fait un modelage du fonctionnement du centre ou d'un objet d'apprentissage ciblé. Toutefois, cette utilisation de l'oral pourrait être considérée comme un enseignement de l'oral seulement si l'apprentissage ciblé est un objet de l'oral. Si ce dernier concerne un objet d'écriture ou de lecture, l'utilisation de l'oral par l'enseignant sera plutôt considérée comme médium d'enseignement, un des deux statuts possibles de l'oral (Lafontaine, 2001).

\footnotetext{
${ }^{2}$ Selon le ministère de l'Éducation de l'Ontario (2015), « les blocs de littératie et de numératie sont des périodes d'apprentissage prioritaires et ne devraient pas être interrompus. »
} 


\section{Les statuts de l'oral}

Dans la classe, l'oral peut avoir deux statuts différents. Il peut être médium d'enseignement ou être objet d'enseignement (Dolz et Schneuwly, 1998; Lafontaine 2001). L'oral est considéré comme médium d'enseignement lorsqu'il est au service des autres volets du français ou des autres disciplines. Ce statut de l'oral est important puisqu'il permet entre autres à l'enseignant de transmettre des explications, de faire un modelage, de questionner les élèves. Il sert aussi à faciliter la communication entre les élèves et l'enseignant (Lafontaine, Plessis-Bélair et Bergeron, 2007). Les élèves utilisent fréquemment l'oral médium pour poser ou répondre à des questions, pour discuter, interpréter, partager leurs opinions, de même que pour résoudre des problèmes ou des conflits. L'oral médium sert aussi aux élèves à verbaliser leurs pensées, leurs réflexions pour ainsi s'approprier, construire et approfondir la compréhension de certaines connaissances (Lentz, 2009).

Bien que l'oral médium soit nécessaire en salle de classe, il est important que l'oral soit également traité comme objet d'enseignement/apprentissage afin de permettre aux élèves une prise en charge de leur compétence à communiquer oralement. L'oral est considéré comme objet d'enseignement/apprentissage, lorsqu'il peut être décomposé en différents éléments qui seront enseignés par les enseignants et appris par les élèves (Dumais 2014). Il existe de nombreux objets d'enseignement/apprentissage ${ }^{3}$, par exemple, le volume, le regard, l'intention de communication, les différentes conduites discursives, etc. C'est parce que chaque objet peut être défini, analysé et mis en pratique par les élèves que ces derniers verront leur compétence à communiquer oralement se développer (Dumais, 2014 ; Maurer, 2001). Cette prise en charge de l'oral par les élèves leur permet de faire des apprentissages durables qui pourront être transférés dans différents contextes (Dolz et Schneuwly, 1998; Dumais, 2014; Lafontaine et Préfontaine, 2007).

\section{Objectifs de recherche}

Puisque des enseignants du primaire (Diller, 2010; Nations et Alonso 2001; Owoki, 2005 ; Swartz et al., 2003) disent utiliser les centres de littératie pour planifier et faciliter l'enseignement de la langue, aussi bien en oral qu'en lecture et écriture, nos objectifs de recherche sont les suivants: 1 - identifier les perceptions de deux enseignantes au sujet de la place de l'oral dans les centres de littératie mis en pratique dans leurs classes ; 2 - décrire l'utilisation de l'oral faite dans les centres d'une classe.

\section{Méthodologie}

Cette section présentera le type de recherche utilisée. Elle fera une brève description de l'échantillon. Ensuite, la collecte des données, ainsi que le déroulement de la recherche seront présentés.

\section{Type de recherche}

Cette recherche qualitative a pour but de comprendre l'utilisation de l'oral dans les centres de littératie pour un groupe en particulier. Elle étudiera les participants dans

\footnotetext{
${ }^{3}$ Dans sa thèse, Dumais (2014) en a répertorié 334, qui ont été regroupés selon dix types.
} 
leur milieu naturel (Fortin 2010). Elle est de type exploratoire (Gauthier, 2009), car elle s'intéresse à un thème peu analysé dans les écrits (Van der Maren, 2004) et a pour but de pallier un tant soit peu le vide théorique concernant l'oral dans les centres de littératie.

\section{Échantillon}

Un échantillonnage par choix raisonné (Fortin, 2010) a été utilisé pour cette recherche, c'est-à-dire que les participants ont été sélectionnés en fonction de critères basés sur l'expérience professionnelle. Dans ce cas-ci, le principal critère de sélection était que les enseignantes devaient utiliser les centres de littératie dans leur classe dans le but de planifier et d'organiser l'enseignement du français. Ainsi, deux enseignantes de deuxième année (Ens.1 et Ens.2) ont été approchées pour participer à cette recherche ainsi que les 22 élèves de l'Ens.1. Ces deux enseignantes travaillent en deuxième année (élèves de 7 ou 8 ans), dans une même école d'une commission scolaire de la région des Laurentides au Québec. Un code alphanumérique leur a été attribué : Ens.1 et Ens.2. L'Ens. 1 est une enseignante qui a 18 années d'expérience, dont trois au $1^{\mathrm{er}}$ cycle du primaire. Elle travaille dans cette école depuis trois ans. L'Ens.2 est une enseignante qui a huit années d'expérience, dont trois années au $1^{\mathrm{er}}$ cycle. Elle travaille dans cette même école depuis maintenant six ans.

\section{Outils et méthode de collecte et d'analyse de données}

Pour répondre au premier objectif de recherche, soit d'identifier les perceptions de deux enseignantes au sujet de la place de l'oral dans les centres de littératie mis en pratique dans leurs classes, la collecte de données a été faite à l'aide d'entrevues. Chacune des participantes a accepté de participer à une entrevue. Ces entrevues étaient de type semi-dirigé (Savoie-Zajc, 2011). Chacune des participantes devait répondre à sept questions. Les entrevues ont duré approximativement 15 minutes.

Pour répondre au second objectif, soit de décrire l'utilisation de l'oral dans les centres de littératie, une deuxième collecte a été effectuée à l'aide d'observations non participantes. Les observations non participantes ont été privilégiées afin que les échanges entre les élèves ne soient pas stimulés par un adulte. Deux outils distincts ont été utilisés : le journal de bord et des enregistrements audios.

L'analyse de contenu des entrevues ainsi que des observations ont été faites selon une logique inductive modérée (Savoie-Zajc, 2011). C'est-à-dire que le cadre conceptuel élaboré dans un premier temps a influencé l'analyse des données, tout en permettant une certaine souplesse. Ainsi, les réponses des enseignantes et les observations ont été analysées en fonction des statuts de l'oral, définis dans le cadre conceptuel.

\section{Déroulement de la recherche}

Cette recherche s'est déroulée sur une période de six semaines consécutives, de la mi-mars à la fin du mois d'avril 2016. Deux collectes de données différentes ont été faites. Lors de la semaine 1 , une première collecte a été réalisée auprès des enseignantes à l'aide d'entrevues semi-dirigées. La deuxième collecte a commencé lors de la deuxième semaine et s'est poursuivie pendant les quatre semaines suivantes. Cette deuxième 
collecte a été faite seulement dans la classe de l'Ens. $1^{4}$. Une première séance d'observation non participante a eu lieu en classe afin d'observer l'enseignante et les élèves. Ces observations se sont poursuivies à raison d'une séance d'observation par semaine d'une durée d'environ 90 minutes chacune, pour les trois semaines suivantes. Ces observations ont permis de trianguler les données obtenues dans les entrevues afin d'établir un premier constat de l'utilisation de l'oral dans les différents centres de littératie dans la classe et par le fait même de corroborer les propos des enseignantes au sujet de la présence de l'oral dans les centres de littératie. À partir de la troisième semaine, et ce, jusqu'à la fin de la collecte, des enregistrements audios des échanges entre les élèves ont également été faits à l'aide de tablettes électroniques afin d'avoir un portrait de la manière dont ils utilisent 1'oral lors des centres de littératie.

\section{Résultats}

La section des résultats est présentée en fonction des deux collectes de données. Les résultats des entrevues des enseignantes permettent de rendre compte de leurs perceptions au sujet de la place de l'oral dans les centres de littératie mis en pratique dans leurs classes. Les résultats des observations non participantes ainsi que des enregistrements audios permettent de décrire l'utilisation de l'oral dans ces centres.

\section{Les perceptions des enseignantes}

Les perceptions des deux enseignantes au sujet de la place de l'oral dans les centres de littératie sont assez convergentes. Lors de l'entrevue, la première question posée visait à vérifier ce que les enseignantes pensaient de l'affirmation disant que les centres de littératie permettent de développer et consolider les apprentissages en lecture, en écriture et en communication orale. Les deux enseignantes étaient en accord. «Ben effectivement, c'est vrai. Peu importe que ce soit en lecture, en écriture, en communication orale, les centres de littératie sont faits en sorte [sic] que les enfants développent ces compétences-là... »(Ens.2)

Ensuite, afin d'en savoir plus sur les centres présents en classe, les enseignantes ont présenté ceux qui avaient été mis en place. Dans les deux classes, huit centres de littératie ont été mis en place :

- le centre de lecture à soi (lecture autonome, avec un livre à leur niveau);

- le centre de lecture à deux (lecture à tour de rôle ou en même temps, d'un même livre, à voix haute, suivi de questions) ;

- le centre d'écriture (écriture de différents types de textes selon les enseignements reçus);

- le centre de lecture pour agir (lecture de différents textes suivie d'une tâche à accomplir, par exemple répondre à des questions, exécuter un bricolage, etc.) ;

- le centre d'étude des mots (activités qui permettent de s'exercer à bien orthographier les mots);

\footnotetext{
${ }^{4}$ L'Ens.2 a dû être retirée de sa classe à la suite d'un accident. C'est pour cette raison que le reste de la collecte des données a été poursuivi seulement dans la classe de l'Ens.1. Toutefois, nous avons conservé son entrevue puisqu'elle avait mis des centres de littératie en place depuis le début de l'année. Il était donc possible de vérifier ses perceptions au sujet de l'oral.
} 
- le centre de lecture fluide et expressive (lecture à voix haute pour augmenter la fluidité);

- le centre de calligraphie (pratique de l'écriture cursive);

- le centre d'informatique (tâches diversifiées en lien avec la lecture et l'écriture).

L'Ens. 1 met aussi en place le centre de variété des mots qui vise à augmenter l'étendue lexicale et à enrichir le vocabulaire, ainsi que le centre d'entraide en écriture, qui permet aux élèves (en dyades) de partager leurs écrits, de les commenter et de les améliorer.

Par la suite, les questions d'entrevue amenaient les enseignantes à identifier les centres où l'oral était utilisé. L'Ens.1 a répondu la lecture à deux, la variété des mots et l'appréciation de la lecture. L'Ens.2 a nommé les centres de lecture fluide et expressive, de lecture à deux, de lecture pour agir et d'informatique.

Lorsqu'elles ont été questionnées sur la présence de l'oral dans ces centres, les deux enseignantes ont surtout parlé de son utilisation au service des tâches proposées, donc de son statut de médium d'enseignement. L'Ens.2 a dit en parlant du centre d'informatique : «... souvent je les place en équipe de deux pour justement faire un travail, donner leur opinion sur quelque chose, donc ils devaient le dire à leur camarade, puis ensuite l'écrire. Ils s'entraident comme ça.» L'Ens.1, en évoquant le centre de lecture à deux, explique l'utilisation que les élèves font de l'oral. «... ils ont besoin de faire des messages clairs, que ça soit compris par l'autre élève, puis faut qu'ils soient capables aussi d'avoir de l'écoute. (...) Ils doivent s'aider, ils doivent poser des questions à l'autre, c'est plus à ce niveau-là que je vais dire d'être capable de s'exprimer clairement, par rapport à l'autre, que l'autre puisse comprendre puis d'avoir une écoute aussi. »

Malgré cette utilisation de l'oral dans ces centres, les deux enseignantes ont été unanimes. Il n'existe pas de centre dans leur classe pour travailler l'oral en tant qu'objet d'enseignement/apprentissage. Bien que certaines informations nous laissent croire que l'oral puisse être objet d'enseignement dans leur façon de le traiter, les enseignantes ne semblent pas en être conscientes. L'Ens. 1 l'exprime concrètement en parlant du centre de variété des mots : "Ça enrichit leur vocabulaire, on apprend aussi la définition, on apprend à l'utiliser dans le bon contexte, en faisant des phrases et tout ça. C'est quand il [l'élève] vient pour s'exprimer oralement, bien c'est ça, il a un vocabulaire plus riche. » Toutefois, son témoignage explique une retombée sur la communication orale, sans que cette dernière soit consciente d'y travailler spécifiquement un objet de l'oral présent dans la Progression des apprentissages (MELS 2011) ${ }^{5}$, c'est-à-dire l'expérimentation de nouveaux mots ou de nouvelles expressions ayant émergé en classe ou issus d'œuvres de littérature de jeunesse.

Aucune des deux enseignantes ne fait de minileçons au sujet de la communication orale. L'Ens.1 ne l'enseigne pas du tout, car c'est une autre enseignante qui se charge de cette portion du français dans sa classe. L'Ens.2, quant à elle, dit l'enseigner, mais pas à l'intérieur des centres de littératie. Toutefois, le fait de se faire poser la question a permis

${ }^{5}$ La Progression des apprentissages est un document officiel produit par le ministère de l'Éducation, du Loisir et du Sport du Québec en 2011 qui présente les connaissances et les stratégies de la compétence à communiquer oralement en fonction des différents niveaux d'enseignement ainsi que leurs contextes d'utilisation. 
à chacune d'elles de réaliser qu'il serait facile de l'intégrer à la planification de quoi? et d'en faire des minileçons. En effet, les deux enseignantes s'entendent pour dire qu'un centre de littératie de l'oral serait intéressant et permettrait de mieux travailler l'oral. L'Ens.1 ajoute que cela lui permettrait de structurer son enseignement. De son côté, l'Ens.2 ajoute qu'à l'aide d'outils technologiques (iPad), les élèves pourraient «s'entendre, s'écouter, puis voir aussi, même se filmer pour voir leur posture...»

\section{La place de l'oral dans les centres de littératie}

Les observations non participantes ayant eu lieu dans la classe de l'Ens.1 ont permis de trianguler les propos recueillis par les enseignantes lors des entrevues, permettant ainsi de constater que l'oral était essentiellement utilisé comme médium d'enseignement. L'Ens.1 a utilisé l'oral principalement lors de ses minileçons au sujet de l'écriture ou de la lecture. Elle a également utilisé l'oral pour expliquer ce qu'elle attendait des élèves au sujet de la tâche proposée. Elle a fait le modelage de certaines stratégies de lecture et a utilisé l'oral pour donner des consignes en ce qui concerne les comportements à adopter et le déroulement de la période. L'oral a facilité la communication entre les différents acteurs. Ainsi, tant l'enseignante que les élèves ont utilisé l'oral pour poser ou répondre à des questions.

De plus, ces observations et les échanges des élèves enregistrés pendant les centres ont permis d'identifier les centres de littératie qui favorisaient l'utilisation de l'oral et de déterminer ses modes d'actualisation. Il s'agit des centres de lecture à soi, d'entraide en écriture, d'informatique, de lecture fluide et expressive ainsi que le centre de lecture à deux. Lors des séances d'observation, même s'il avait été possible de voir une utilisation de l'oral (selon les dires de l'Ens.1), les centres de littératie d'étude des mots, de lecture pour agir, d'écriture, de calligraphie ainsi que le centre de variété des mots n'ont pas permis de constater une utilisation de l'oral. Ils ne seront donc pas commentés ici. Nous commenterons seulement les centres où l'oral a été observé.

Centre de lecture à soi. Dans ce centre, chacun des enfants doit lire de manière autonome en silence. Pour trois des quatre séances d'observation, la présence de l'oral n'a pas pu être observée. Toutefois, lors d'une séance, l'enseignante a terminé la période en donnant la parole aux enfants afin qu'ils puissent parler du personnage principal de leur livre. Ainsi, ces derniers ont utilisé l'oral afin d'échanger sur les caractéristiques du personnage principal. D'une intervention à l'autre, il a été possible d'observer l'évolution de la compréhension des élèves au sujet des caractéristiques du personnage principal. En effet, ces derniers réutilisaient les propos intéressants des uns et des autres afin de partager une vision plus définie des caractéristiques de leur personnage.

Centre d'entraide en écriture. Ce centre de littératie a permis aux élèves de lire leur texte à un coéquipier pour ensuite recevoir des suggestions de ce dernier. Les élèves devaient donc être à l'écoute de ce qui était lu pour ensuite y réagir en faisant des propositions diverses. Ces propositions peuvent concerner la cohérence du texte, la syntaxe, la ponctuation, les accords, l'orthographe des mots et le choix des mots, comme 
le montre cet extrait entre Martin et $\mathrm{Vynce}^{6}:$ « $:$ La petite fille a une glace sur la tête parce qu'elle est malade. - M : On pourrait dire au lieu d'une glace une bouillotte. »

Ainsi dans cet extrait, nous pouvons constater que Martin écoute attentivement la phrase de Vynce, tout en gardant en tête l'intention de ce centre, c'est-à-dire s'entraider afin d'améliorer les textes écrits. Il propose un choix de mot différent à Vynce. Bien que ce dernier ne réagisse pas à l'oral, le fait qu'il choisisse d'effacer son mot pour le remplacer par la suggestion de Martin démontre la prise en compte de l'opinion de son coéquipier.

Centre d'informatique. La tâche proposée et observée concernait la grammaire. En équipe de deux, les élèves devaient repérer et souligner les noms présents dans quelques phrases. Ils devaient ensuite déterminer le genre et le nombre pour chacun des noms. Les observations ont permis de relever que les élèves utilisaient l'oral pour s'entraider, mais aussi pour argumenter lorsqu'ils n'étaient pas d'accord. Ils parvenaient à échanger différents points de vue et à justifier leur position en se référant à leurs connaissances grammaticales.

Centre de lecture fluide et expressive. Ce centre a permis de faire de nombreuses observations sur l'oral. En premier lieu, les élèves tentaient de lire un texte à voix haute et d'en faire une lecture fluide et expressive. Les textes proposés pouvaient être des poésies, des extraits de pièce de théâtre pour enfant ou des textes comportant certaines difficultés. Dans le cas observé, il s'agissait d'un extrait de pièce de théâtre. Puisque les élèves devaient produire une lecture collective, ils se sont permis de commenter la manière de lire des uns et des autres. Chacun d'entre eux s'est assuré que les coéquipiers avaient utilisé la bonne intonation et qu'ils avaient mis suffisamment d'expression dans leur lecture. Ils n'ont pas hésité à s'interrompre, à se reprendre et à s'exercer de manière répétitive. Sans en être conscients, les élèves eux-mêmes ont mis en évidence des objets de l'oral. Il a été possible d'observer des interventions des élèves concernant des éléments prosodiques tels que le débit, la prononciation ainsi que l'intonation. Puisqu'aucun enseignement spécifique n'a été fait concernant ces objets de l'oral, il est possible de croire que les élèves faisaient référence à leurs connaissances antérieures de ces objets.

De plus, pour arriver à une lecture fluide et expressive, les élèves devaient bien comprendre le sens de ce qu'ils lisaient. Il a donc été possible d'observer des élèves qui se questionnaient entre eux sur la signification de certains mots ou de certaines expressions.

A : «Nous sommes des chiens savants. Nous savons parler mathématique.

L : André, est-ce que tu sais qu'est-ce que c'est? Est-ce que tu sais qu'est-ce que ça veut dire "on sait parler mathématique"?

A : C'est des maths, c'est des plus...

L : On peut parler mathématique... on peut parler mathématique... Tu sais ce que ça veut dire?

${ }^{6}$ Pour préserver l'anonymat des élèves, les prénoms ont été changés. 
$\mathrm{K}$ : Toi Léa, tu sais-tu qu'est-ce ça veut dire?

L: Non.

A : Il y a dedans le verbe mather.

$\mathrm{L}$ : Ouais. C'est en math.

$\mathrm{K}$ : J'ai déjà entendu ce mot-là pis je me souviens pu qu'est-ce ça veut dire.

L : Je sais pas là.

A : Ça se peut que c'est des personnes qui sont des chiens.

L : Ouais, ça aussi... Peut-être qu'ils sont bons en mathématiques, je le sais pas.

$\mathrm{K}$ : P'têtre qu'ils voulaient dire que... mathématique, mathématique comme...

L : Attends... Moi je dis mathématiques, pis là... ok on reviendra après là.

$\mathrm{K}$ : Ok peut-être que ça va le dire. »

Dans cet extrait, Léa exerce sa compréhension orale avec deux coéquipiers. Son écoute est attentive et, lorsqu'André utilise une expression inhabituelle, elle l'arrête et le questionne sur le sens de l'expression «parler mathématique ». Elle réfléchit à la réponse proposée par André, sans en être satisfaite. André tente de trouver le sens en utilisant une stratégie qu'il connait, c'est-à-dire trouver un petit mot dans un grand mot. Dans ce casci, cette stratégie n'est pas efficace. Après quelques propos qui ne permettent pas à Léa de mieux comprendre, cette dernière suggère une autre stratégie, c'est-à-dire continuer la lecture. Katia comprend finalement la suggestion. En disant : «Ok, peut-être que ça va le dire », elle précise le but de cette stratégie qui est de continuer la lecture afin de vérifier si des informations nouvelles nous permettront de mieux comprendre.

Centre de lecture à deux. Dans ce centre, l'oral occupe une place importante. Tout d'abord, les élèves ont fait une lecture oralisée, une page à la fois, à tour de rôle d'un même livre. Ensuite, au bout de chaque page, les élèves se posaient des questions qui provenaient d'une banque de questions générales qui concernaient les quatre dimensions de la lecture, c'est-à-dire des questions de compréhension, d'interprétation, de réaction et d'appréciation. Selon les observations, certains élèves semblaient coincés par cette banque et cherchaient à poser la bonne question, alors que d'autres posaient des questions spontanées et semblaient plus à l'aise de modifier les questions en fonction du texte lu. Les observations ont aussi démontré que les élèves ont utilisé l'oral pour s'entraider. Par exemple, il a été possible de les observer s'interrompre pour demander à leur coéquipier de reprendre un mot ou une phrase qui n'avait pas été bien lue. Les observations ont aussi permis de constater que certains enfants ont offert les bonnes réponses à leur coéquipier, lorsque ce dernier était en difficulté. D'un autre côté, il a été possible de constater aussi que certains élèves ont plutôt questionné leur coéquipier afin de lui permettre de trouver lui-même les stratégies possibles à utiliser. C'est également dans ce centre que les élèves discutent le plus de leur compréhension du texte et se questionnent énormément sur le sens des mots et des expressions, comme le présentent les différents extraits suivants.

$\mathrm{T}:$ «Est-ce que tu sais c'est quoi un Valentin?

L: Oui.

M : Moi aussi.

L : C'est quoi? 
T : Ben c'est, j'pense que c'est à Saint-Valentin pis c't'un genre de bonhomme tout nu.

Ben pas tout nu mais $/^{7}$

L : Non, c'est un garçon/

$\mathrm{T}:$ Ben c'est ça/

L : Admettons qu't'aurais une blonde. Exemple.

$\mathrm{T}: \mathrm{J}$ 'en ai pas moi.

L : Admettons qu't'en aurais une. Depuis longtemps. Ben tu serais un Valentin. Le jour de la St-Valentin ta blonde ça serait ta Valentine pis toi tu s'rais son Valentin. Tu comprends? C'est un amoureux.

$\mathrm{T}$ : Oh un amoureux. J'pensais pas que c'tait ça ».

Dans cet extrait, on peut constater que Tim veut s'assurer que Léa comprend bien le sens du mot Valentin tout en démontrant que lui aussi en connait la signification. Cette dernière connait la signification du mot, mais propose plutôt à Tim de partager son opinion en premier. Elle explique ensuite sa définition du mot, ce qui permet à Tim de prendre conscience des différences entre les deux définitions.

L'extrait suivant présente un échange entre Audrey et Marianne qui se questionnent sur le sens d'un mot.

A : (lecture)... tu prendras bien un cuissot de dino ?

$\mathrm{M}$ : Audrey toi tu sais ce que ça veut dire quoi un cuissot?

A : Oui, parce que des cuisses tsé là des cuisses de poulet, ça dit cuissss — peut-être que c'est une cuisse de dinosaure.

M : Pis en même temps l'image est-ce qu'elle te donne une même idée?

A : Oui parce que, regarde, il mange ça. Pis je pense pas qu'une cuisse de poulet ça soit gros comme ça. J'en mange moi des cuisses de poulet.

On peut constater dans cet extrait qu'Audrey utilise au départ une stratégie lexicale pour fournir la signification du mot cuissot. Marianne cherche à appuyer cette explication par l'utilisation d'une autre stratégie pour confirmer le sens, soit de regarder l'image. Audrey confirme et fait des liens avec sa vie pour justifier son hypothèse.

L'extrait suivant présente un échange où Katia supervise la lecture d'Ariane en lui proposant différentes stratégies.

$\mathrm{K}$ : Ariane, lis moins vite, ça va t'aider à lire.

A : Ok oui.

A : Est-ce que tu sais qu'est-ce que ça veut dire un châ-tai-gnier?

$\mathrm{K}$ : Châtaignier? Heu pas vraiment non. Je le sais pas mais on pourrait regarder s'il y a un mot qu'on connait dedans le mot.

A : Châtaignier ça m'fait penser à chêne. Fait que j'pense plus que c'est une sorte d'arbre.

$\mathrm{K}$ : On va recommencer la phrase fait que peut-être que ça va nous aider.

\footnotetext{
${ }^{7}$ Dans cet extrait, la barre oblique signifie que les élèves se font couper la parole.
} 
A : (Relit la phrase... regarder les feuilles de châtaignier...) Ah c't'un arbre, c't'un arbre.

Katia verbalise très bien les stratégies qu'il est possible d'utiliser pour mieux comprendre le sens d'un mot inconnu. On voit dans cet échange que Katia supervise bien la lecture d'Ariane. Elle lui suggère de lire moins vite et lorsqu'Ariane se questionne sur le sens d'un mot, Katia lui suggère d'utiliser certaines stratégies. Lorsqu'Ariane exprime que le mot lui fait penser à "chêne », Katia ne la contredit pas, mais ne semble pas convaincue non plus, car elle lui propose une autre stratégie.

\section{Discussion}

Cette recherche a permis d'identifier des perceptions similaires chez les deux enseignantes en ce qui concerne la place de l'oral dans les centres de littératie. En effet, l'oral aurait une grande place dans ces centres, mais il n'y serait pas enseigné spécifiquement. En instaurant un centre de littératie de l'oral dans leur classe, les enseignantes affirment qu'il serait beaucoup plus facile de structurer l'enseignement de l'oral et de l'enseigner réellement. Effectivement, à l'aide d'un centre de littératie de l'oral, il serait possible de considérer l'oral comme un objet d'enseignement/ apprentissage. En planifiant l'enseignement de différents objets de l'oral, les enseignantes pourraient mieux définir certains objets de l'oral pour les élèves et leur permettre ainsi de les analyser et de les mettre en pratique. Et comme le soulignent certains chercheurs, c'est en devenant conscient des objets de l'oral et en prenant en charge le développement de leur compétence que les élèves pourront faire des apprentissages durables et transférables (Dolz et Schneuwly, 1998; Dumais, 2014; Lafontaine et Préfontaine, 2007).

Cette recherche a aussi permis, grâce aux séances d'observation non participantes ainsi qu'aux enregistrements audios, de décrire l'utilisation de l'oral dans ces centres. L'Ens. 1 a utilisé l'oral à maintes reprises. C'est un oral médium d'enseignement qui a été utilisé par cette dernière. En effet, la présence de l'oral a été perçue lors des minileçons concernant la lecture et l'écriture, ainsi que dans la formulation de consignes ou de réponses données aux élèves. Aucun enseignement spécifique de l'oral n'a été observé. Ainsi, tout au long des observations, l'oral a gardé son statut de médium d'enseignement. Il ne semble donc pas y avoir d'enseignement de l'oral dans les centres de littératie mis en pratique par cette enseignante.

Du côté des élèves, l'oral a été utilisé fréquemment aussi, mais toujours au service de l'écriture ou de la lecture. Les élèves ont surtout utilisé l'oral pour approfondir leur compréhension des textes lus, pour se faire des suggestions entre eux et aussi pour défendre leur point de vue. En mettant en place un centre de littératie de l'oral, plusieurs objets de l'oral pourraient être enseignés et mis en pratique afin de soutenir les élèves dans les activités proposées dans les centres de littératie. Par exemple, l'écoute est nécessaire dans tous les centres où l'oral est présent. Des stratégies d'écoute pourraient être enseignées pour ainsi permettre d'adopter une attitude d'ouverture, d'utiliser des conduites qui favorisent la communication, de témoigner de son écoute en reformulant ce qui a été dit. Aussi, afin de favoriser des réactions intéressantes, il serait pertinent d'enseigner aux élèves à utiliser certains procédés discursifs tels que la justification afin d'apprendre à faire valoir son point de vue lorsqu'il y a une divergence d'opinions quant 
à la réponse attendue ou au déroulement d'une tâche présente dans un centre de littératie. L'enseignement de différents actes de parole, par exemple, exprimer son désaccord, pourrait être nécessaire afin que les élèves puissent l'utiliser, par exemple dans le centre d'entraide de l'écriture, lorsque des propositions ne sont pas jugées adéquates. Aussi, la compréhension de ce que sont les intentions de communication pourrait s'avérer un élément intéressant à enseigner afin d'éviter les propos hors sujet. Il ne s'agit ici que de quelques exemples d'objets d'oral possibles à enseigner pour rendre le travail en centre de littératie plus efficace. Toutefois, il semble important que l'enseignement de l'oral dans un centre de littératie de l'oral n'ait pas pour seul objectif de rendre les élèves efficaces dans les échanges qu'ils ont entre eux lors des centres de littératie. L'oral est au cœur des interactions sociales. Ainsi l'enseignement d'objets d'apprentissage de l'oral à l'intérieur d'un centre de littératie de l'oral doit aussi avoir comme visée le développement et la mise en pratique des habiletés du volet oral de la littératie.

Un centre de littératie de l'oral permettrait possiblement aux enseignantes de récupérer le temps qui semble leur manquer pour l'enseignement de l'oral. En effet, en intégrant l'enseignement de l'oral dans ce dispositif de gestion de l'enseignement de la langue (Kracl, 2012), il est possible de croire que les enseignants arriveront à mieux structurer l'enseignement et l'apprentissage de l'oral, en planifiant des minileçons sur des objets spécifiques et en proposant des activités concrètes où chacun aura la possibilité de s'exercer. De plus, un accompagnement auprès des enseignants semblerait propice. L'accompagnement est de plus en plus considéré pour la formation continue (Carraud, 2010 ; Paul, 2009 ; Roquet, 2009). Cela permettrait aux enseignants qui manquent parfois de connaissance sur les objets spécifiques de l'oral (Dumais, 2014) de mieux comprendre ce dont il est question et de mieux structurer l'enseignement de l'oral.

\section{Conclusion}

L'objectif de cette recherche était de décrire la place de l'oral dans les centres de littératie. À l'aide d'entrevues demi-dirigées, d'observations non participantes et d'enregistrements, il a été possible de constater que l'oral a un statut de médium (Lafontaine 2001). Il a été utilisé par les enseignantes dans le but d'expliquer, de donner des consignes, de faire le modelage de certaines stratégies ainsi que de répondre aux questions. Du côté des élèves, l'oral a été utilisé pour poser des questions, réfléchir à voix haute et s'entraider. Contrairement à ce que certains professionnels de l'enseignement affirment, il n'a pas été possible de confirmer que les centres de littératie permettent une organisation efficace de l'enseignement et des apprentissages en ce qui concerne l'oral. Pour y parvenir, il faudrait que l'oral ait un statut d'objet d'enseignement/apprentissage, c'est-à-dire qu'il puisse être observé, analysé et mis en pratique par les élèves (Dumais, 2014; Maurer, 2001). Aussi, cette recherche a permis de poser un premier regard sur l'oral au sein de centres de littératie du primaire. Elle contribue, dans une certaine mesure, à faire avancer les connaissances à ce sujet, et ce, malgré quelques limites. D'abord, les entrevues rapportent seulement les perceptions de deux enseignantes. De plus, les observations ont été faites dans la classe d'une seule enseignante. Également, cette recherche s'est effectuée dans une seule école et il est probable que les deux enseignantes aient développé une vision commune en raison du travail d'équipe et des discussions qui s'en suivent. Ainsi, en effectuant des observations dans différentes écoles, il serait possible d'avoir une meilleure vue d'ensemble de la place de l'oral au sein des 
centres de littératie. D'autres recherches seront nécessaires pour continuer à décrire l'utilisation de l'oral dans les centres de littératie, afin de constater ce qui se fait à ce sujet dans différents milieux et à différents niveaux scolaires, ce qui permettrait de confirmer les conclusions de cette recherche. De plus, des recherches-actions ou des recherches collaboratives seraient nécessaires afin de développer un centre de littératie de l'oral pour permettre un réel enseignement et apprentissage des objets de l'oral ainsi qu'un accompagnement adéquat des enseignants, afin de permettre un changement des pratiques en enseignement de l'oral. Aussi, il serait bien de déterminer quelles approches didactiques de l'oral (oral par les genres, oral pragmatique, oral intégré) (Dumais et al., accepté) seraient les plus appropriées pour s'intégrer à ce dispositif d'enseignementapprentissage.

\section{Références}

Bianco, M. (2015). Du langage oral à la compréhension de l'écrit. Grenoble : Presses universitaires de Grenoble.

Buors, P., \& Lentz, F. (2009). Les littératies multiples : un cadre de référence pour penser 1 ' intervention pédagogique en milieu francophone minoritaire. Cahiers FrancoCanadiens de l'Ouest, 21, 127-150.

Carraud, F. (2010). Faut-il être accompagné pour apprendre à enseigner ? Recherche et Formation, 63, 121-131.

Conseil scolaire catholique du Nouvel-Ontario. (2012). Programme pédagogique et temps d'apprentissage. Repéré à http://docs.nouvelon.ca/doc/DA/ELV03_04.pdf.

Diller, D. (2010). Les centres de littératie: susciter l'engagement des élèves en lecture, en écriture et en communication orale. Montréal : Chenelière Éducation.

Dolz, J., \& Schneuwly, B. (1998). Pour un enseignement de l'oral : initiation aux genres formels à l'école. Issy-les-Moulineaux : ESF éditeur.

Dumais, C. (2014). Taxonomie du développement de la langue orale et typologie : fondements pour l'élaboration d'une progression des objets

d'enseignement/apprentissage de l'oral en classe de français langue première qui s'appuie sur le développement. Thèse de doctorat inédite, Université du Québec à Montréal.

Dumais, C, (2015). Une typologie des objets de l'oral pour la formation initiale et continue des enseignants, in R, Bergeron, C. Dumais, B. Harvey, \& R. Nolin, (Eds.), La didactique du français oral du primaire à l'université. (pp. 27-52). Montréal : Éditions Peisaj.

Dumais, C., Soucy, E. \& Plessis-Bélair, G. (à paraitre). La didactique de l'oral au préscolaire et au primaire au Québec : portrait d'un domaine de recherche en émergence, in S. El Euch, A. Groleau, \& Samson, G. (Eds.) Les didactiques : bilans et perspectives. Québec : Presses de l'Université du Québec.

Dumais, C. \& Lafontaine, L. (2011) L'oral à l'école québécoise: portrait des recherches actuelles. Revue suisse des sciences de l'éducation, 33 (2) 2011, 285-302.

Flemington, K., Hart-Hewins, L., Proulx, J., \& Villier, U. (2012). Premiers pas en littératie : des centres de littératie dès la maternelle. Montréal : Modulo.

Ford, M. P., \& Opitz, M. F. (2002). Using Centers to Engage Children During Guided Reading Time The Reading Teacher, 55 (8), 710--717. 
Fortin, M.-F. (2010). Fondements et étapes du processus de recherche : méthodes quantitatives et qualitatives. Montréal: Chenelière Éducation.

Fountas, I. C., \& Pinnell, G. S. (1996). Guided Reading. Good first teaching for all children. Portsmouth: Heinemann.

Gauthier, B. (2009). La structure de la preuve, in B. Gauthier (Eds.), Recherche sociale: de la problématique à la collecte des données (pp. 169-198). Sainte-Foy, Québec : Presses de l'Université du Québec.

Hodges, T. S., \& McTigue, E. M. (2014). Renovating Literacy Centers for Middle Grades: Differentiating, Reteaching, and Motivating. The Clearing House: A Journal of Educational Strategies, Issues and Ideas, 87 (4), 155-160.

Kracl, C. (2012). Using Literacy Stations to Manage Small Group Instruction. International Journal of Humanities and Social Science, 2(18), 200-209.

Lafontaine, L. (2001). Élaboration d'un modèle didactique de la production orale en classe de français langue maternelle au secondaire. Thèse de doctorat inédite. Montréal : Université du Québec à Montréal.

Lafontaine, L. \& Pharand, J. (2015). La littératie un concept en évolution, in L. Lafontaine, \& J. Pharand, (Eds), Littératie : vers une maîtrise des compétences dans divers environnement (pp. 1-11). Québec: Presses de l’Université du Québec.

Lafontaine, L. Plessis-Bélair, G. \& Bergeron, R. (2007). La didactique du français oral au Québec : un historique, quelques réflexions et des questions actuelles, in G. PlessisBélair, L. Lafontaine \& R. Bergeron, (Eds.) La didactique du français oral au Québec. (pp.1-36). Québec: Presses de l'Université du Québec.

Lafontaine, L. \& Préfontaine, C. (2007). Modèle didactique descriptif de la production orale en classe de français langue première au secondaire. Revue Des Sciences de L'éducation, 33(1), 47-66.

Lentz, F. (2009). L'oral, pour se dire : remarques sur la communication orale dans les apprentissages en français en milieu francophone minoritaire. Cahiers FrancoCanadiens de l'Ouest, 21(1-2), 211-227.

Maurer, B. (2001). Une didactique de l'oral : du primaire au lycée. Paris : BertrandLacoste.

Ministère de l'Éducation, du Loisir et du Sport du Québec (2011). Progression des apprentissages au primaire. Français, langue d'enseignement. Québec : Gouvernement du Québec.

Nations, S., \& Alonso, M. (2001). Primary Literacy Centers. Gainesville: Maupin House. Owocki, G. (2005). Time for Literacy Centers. Portsmouth: Heinemann.

Paul, M. (2009). Accompagnement. Recherche et Formation, 62, 91-107.

Roquet, P. (2009). L'Émergence de l'accompagnement. Une nouvelle dimension de la formation. Recherche et Formation, 62, 13-24.

Rosselle, M. (2013). Les MOOC: des dispositifs d'enseignement-apprentissage à personnaliser. Document inédit. Toulouse: Atelier MOOC, EIAH. Repéré à file:///C:/Users/morean01/Downloads/Les\%20MOOC_des\%20dispositifs\%20d\%2527ensei gnement-apprentissage $\% 20 \%$ C3\%A0\%20personnaliser\%20\%25281\%2529.pdf

Russel, D. (2014). Fostering Third Graders'Attributes of Indepndence During the Literacy Block: A Self Study. The college at Brockport: State University of NewYork. Brockport, New-York 
Rybczynski, M., \& Troy, A. (1995). Literacy-Enriched Play Centers: Trying Them Out in "The Real World". Childhood Education, 72(1), 7-12.

Savoie-Zajc, L. (2011). La recherche qualitative/interprétative en éducation, in $\mathrm{T}$. Karsenti, T. \& L. Savoie Zajc, (Eds.), La recherche en éducation : étapes et approches. Saint-Laurent, Québec: ERPI.

Sénéchal, K. \& Chartrand, S,G, (2012). Représentations et pratiques de l'enseignement de l'oral en classe de français : changements et constantes depuis 25 ans, in R. Bergeron, \& G. Plessis-Bélair, (Eds.), Représentations, analyses et descriptions du français oral, de son utilisation et de son enseignement au primaire, au secondaire et à l'université (pp.185-199). Montréal : Éditions Peisaj.

Simard, C., Dufays, J.L., Garcia-Debanc, C. \& Dolz J. (2010). Didactique du français langue première. Bruxelles: De Boeck.

Soucy, E. (2016). Les centres de littératie, un dispositif qui pourrait permettre l'enseignement de l'oral. Communication présentée au $84^{\mathrm{e}}$ Congrès de l'ACFAS, colloque «L'oral et sa didactique : quels nouveaux enjeux? », Montréal, Canada.

Stone, S. J. (1996). Teaching Strategies: Promoting Literacy Through Centers. Childhood Education, 72(4), 240-241.

Stout, R. (2009). Putting literacy centers to work: a novice teacher utilizes literacy centers to improve reading instruction. Networks an on-line journal for teacher research, 11, (1), 1-6.

Swanson, M. (2013). The effects of choice on young children's reading engagement and stamina. Mémoire de maitrise.University of Wisconsin-River Falls. River Falls, Wisconsin.

Swartz, S. L., Shook, R. E., Klein, A. F., Moon, C., Bunnell, K., Berlt, M., \& Huntley, C. (2003). Guided Reading \& Literacy Centers. California: Dominie Press, Inc.

Van der Maren, J-M. (2004). Méthodes de recherche pour l'éducation. Bruxelles : De Boeck.

Worthy, J., Maloch, B., Pursley, B., Hungerford-Kresser, H., Hampton, A., Jordan, M., \& Semingson, P. (2015). What Are the Rest of the student doing? Language Arts, 92(3), 173-186.

Biographie de l'auteur

Emmanuelle Soucy est doctorante en science de l'éducation à l'Université du Québec en Outaouais. Ses intérêts de recherche portent sur la didactique du français au primaire. Dans le cadre de sa thèse, elle s'intéresse particulièrement aux centres de littératie et à l'enseignement de l'oral. Elle est enseignante au primaire depuis de nombreuses années. Elle est également chargée de cours, superviseure de stage et assistante de recherche. 